\title{
Could SARS-CoV-2 Infection Change Fetal Cardiac Output?
}

\author{
Ezgi Turgut ${ }^{1}$, Bedri Sakcak ${ }^{1}$, D.UYAN HENDEM ${ }^{1}$, Deniz Oluklu ${ }^{1}$, Sule Goncu Ayhan ${ }^{1}$, \\ and Dilek Sahin ${ }^{1}$ \\ ${ }^{1}$ Division of Perinatology Department of Obstetrics and Gynecology Ministry of Health \\ Ankara City Hospital Ankara Turkey
}

March 6, 2022

\begin{abstract}
Aim: We aimed to examine fetal cardiac output $(\mathrm{CO})$ in patients who recovered from severe acute respiratory syndrome coronavirus 2 (SARS-CoV-2) infection. Materials: This prospective study included 48 pregnant women recovered from SARS$\mathrm{CoV}-2$ infection and 50 control cases. SARS-CoV-2 infection was diagnosed by polymerase chain reaction (PCR) test in patients. Fetal echocardiographic evaluations were performed at 24-37 weeks of gestation in pregnant women who recovered from the infection and control group. Results: The median value of ultrasound evaluation was 34 (2.6) weeks of gestation in the recovery from the SARS-CoV-2 infection (RSI) group, and 32 (7.6) weeks in the control group ( $\mathrm{p}=0.565)$. Left cardiac output (LCO) $\mathrm{z}$ score was significantly lower in the RSI group than the control group $(\mathrm{p}=0,041)$. LCO and combine cardiac output (CCO) $\mathrm{z}$ score were significantly lower in the severe disease group than mild, moderate disease groups, and controls ( $\mathrm{p}=0,019$ and $\mathrm{p}=0,013)$. CCO $(\mathrm{mL} / \mathrm{min} / \mathrm{kg})$ was decreased in the severe disease group when compared with control and mild disease groups $(\mathrm{p}=0,044)$. Fetal distress, preterm delivery rate, and neonatal intensive care unit (NICU) admission were found to be higher in the severe disease group compared to the control group $(\mathrm{p}=0,010, \mathrm{p}=0,009$, and $\mathrm{p}<0,001$ respectively). Conclusion: In the present study, fetal cardiac output in pregnant women with recovery from SARS-CoV-2 infection was found significantly decreased, especially in whom had severe diseases. Placental dysfunction and inflammatory cytokines might cause fetal cardiac changes. Further studies could be clarified on the impact of SARS-CoV-2 infection on fetal cardiac function.
\end{abstract}

\section{Introduction}

Severe acute respiratory syndrome coronavirus 2 (SARS-CoV-2) infection is a major public health problem, with hospitalizations, admissions to the intensive care unit (ICU), and even deaths Compared with nonpregnant women of childbearing age with SARS-CoV-2 infection, pregnant women are more likely to be needed for respiratory support and admitted to an ICU [2]. Pregnant women with SARS-CoV-2 infection are at high risk for adverse perinatal outcomes such as early pregnancy loss, fetal growth retardation, and preterm delivery $[3,4]$.

Impaired placental function, hypoperfusion, and inflammation might lead to fetal decompensation that increased risk of perinatal mortality and morbidity $[5,6]$.

Cardiac output depends on heart rate, preload, afterload and myocardial contractility . An increase in afterload or a decrease in preload causes a decrease in cardiac output Changes in cardiac output have been demonstrated in hydrops fetalis, fetal growth retardation (FGR), anemia, and various pathological conditions . In this present study, we aimed to evaluate changes of fetal cardiac output in pregnant women who recovered from SARS-Cov-2 infection.

\section{Material-Method}

This prospective study included 48 pregnant women with recovered from SARS-CoV-2 infection and 50 control cases. Approval for the study was obtained from Ankara City Hospital Ethics Committee with 
the decision number E2-21-639. Written consent was obtained from patients. SARS-CoV-2 infection was diagnosed by polymerase chain reaction (PCR) test in patients. Twin pregnancy, maternal systemic disease, fetal anomaly, and aneuploidy were excluded from the study. Fetal echocardiographic evaluations were performed between 24-37 weeks of gestation by the same maternal-fetal medicine specialist using C1-5RS convex probe (1.75-4.95 Mhz) GE Voluson S10 Ultrasound. The study group and control group were matched for maternal and gestational age. A two-dimensional assessment of the great vessels and Doppler flow interrogation was obtained according to the guidelines of the International Society of Ultrasound in Obstetrics and Gynecology [14]. Aortic (AV) (figure 1) and pulmonary artery valve (PV) (figure 2) diameters were measured in systole [14]. Power Doppler cursor was located in parallel to the long axis of the aorta or pulmonary artery immediately distal to the valves. The angle between the ultrasound cursor and the direction of blood flow was $<10^{\circ}$. The pulmonary and aortic valves flow velocity waveforms were obtained from the left and right ventricular outflow tract views (Figure 3) [15]. Velocity time integral (VTI) and heart rate (HR) were measured and averaged over the three best cardiac cycles. Cardiac outputs for the left and right ventricle (LCO and RCO) were calculated separately as VTI $\times \pi($ AV or PV diameter/2)2 $\times$ HR [15]. Gestational age was determined using first-trimester head-rump length. LCO and RCO z scores were calculated according to the gestational week. Estimated fetal weight (EFW) was calculated with the method of Hadlock et al. Combined cardiac output $(\mathrm{CCO}=\mathrm{RCO}+\mathrm{LCO})$ was calculated, z score was obtained according to the gestational week $[16,17]$. Also, CCO was normalized by estimated fetal weight [18]. Demographic and echocardiographic data were compared between recovery from the SARS-CoV-2 infection (RSI) and control groups. Patients in the study group were divided into subgroups according to the World Health Organization (WHO)'s disease severity classification [19]. Echocardiographic and perinatal outcomes were compared between these subgroups.

Descriptive statistics including the mean, standard deviation or median, and minimum-maximum values for numerical measures were calculated for all patients. The normality of the variables was tested with both Shapiro - Wilk and Kolmogorov - Smirnov tests. Two groups were compared with The Student's t-test and Mann-Whitney U test. One Way ANOVA analysis (and post hoc test to compare groups in case of significant difference) and Kruskal Wallis test, Mann- Whitney U-test with Bonferroni correction were used to compare the groups. For categorical variables, a comparison of variables was performed by Pearson Chi-square test and Fisher's exact test. The alpha significance level of 0.05 was used to assess statistical significance. Statistical analysis was done with IBM SPSS Statistics 17.0 (IBM Corporation, Armonk, NY, USA).

\section{Results}

The demographic data of the study population was shown in Table 1 and the maternal baseline characteristics were similar in both groups ( $>0,05)$. In table 2, the clinical points of SARS-CoV-2 infection were shown and the gestational age of the SARS-CoV-2 infection diagnosis was 20 (8-34). In the RSI group, 18 (37.5\%) patient had mild disease, $20(41.6 \%)$ patient had moderate disease, and $10(20.8 \%)$ patient had severe disease. There was no critical patient in this group. Thirty $(62,5 \%)$ of the study patients were hospitalized and $10(20,8 \%)$ of them needed respiratory support. The echocardiographic findings were given in Table 3. All fetuses had normal cardiac morphology. The median value of ultrasound evaluation was 34 (2.6) weeks of gestation in the RSI group, and 32 (7.6) weeks in the control group ( $\mathrm{p}=0.565)$. LCO z score was found significantly lower in the RSI group than the control group $(\mathrm{p}=0,041)$. Table 4 shows the relation between SARS-CoV-2 infection severity and fetal echocardiographic findings. LCO and CCO z scores were significantly lower in the severe disease group than mild disease, moderate disease, and control groups $(\mathrm{p}=0,019$ and $\mathrm{p}=0,013)$. CCO $(\mathrm{mL} / \mathrm{min} / \mathrm{kg})$ was found to be decreased in the severe disease group than mild disease and control groups $(\mathrm{p}=0,044)$. Perinatal outcomes were given in Table 5 . Gestational age at birth and birth weight were significantly lower in the severe disease group compared to mild disease, moderate disease, and control groups $(\mathrm{p}<0,001$ and $\mathrm{p}=0,020)$. Fetal distress, preterm delivery rate, and neonatal intensive care unit (NICU) admission were higher in the severe disease group than controls $(p=0,010, p=0,009$, and $\mathrm{p}<0,001$ respectively).

\section{Discussion}


In this study, we found low LCO z scores in the RSI group than the controls. Also, LCO and CCO z scores were found to be lower in the severe disease group when we divided the RSI group according to the disease severity. Additionally, adverse pregnancy outcomes including fetal distress, preterm delivery rate, and NICU admission were increased in the severe disease group.

The SARS-CoV-2 infection causes an excessive inflammatory response with the release of a large number of proinflammatory cytokines [20,21]. Placental injury and deterioration of feto-maternal perfusion is triggered by inflammation and this have been shown in literature before [20,22]. Furthermore, this proinflammatory event might affect fetal renin-angiotensin system (RAS) that regulates the uteroplacental blood flow by balancing vasodilator and vasoconstrictive pathways [23]. Down-regulation of RAS also leads to impaired uteroplacental blood flow and might be associated with fetal decompensation and adverse pregnancy outcomes. Fetal echocardiography studies may help demonstrate this fetal deterioration [5]. The potential effects of SARS-CoV infection to the prenatal CO have not been previously studied. In this study, we evaluated fetal cardiac output in pregnant women who recovered from SARS-Cov-2 infection and we detected negative changes especially in the severe disease group.

Fetal cardiac output has been evaluated in several studies in obstetric conditions associated with cardiac dysfunction, such as hydrops fetalis, FGR, twin to twin transfusion syndrome (TTTS), and diabetes mellitus (DM) [9-13]. It has been observed that direct calculation of cardiac output could give valuable information for heart failure and is potentially useful in the assessment of fetal well-being [24].

Cardiac output is dependent on preload (circulatory volume), afterload (circulatory resistance), and myocardial contractility [25]. Fetal teratoma, placental chorioangioma, and fetal anemia are known to be associated with high fetal CO as a result of increased preload $[11,26,27]$. Rizzo et al. found that left CO increases in FGR fetuses probably due to cerebral vasodilatation and consequently decreased cardiac afterload [28]. In our study, a decrease in the left CO z score in the RSI group was observed. In addition, we found a significantly lower left $\mathrm{CO}$ z score in the severe disease group than in the other groups. Our findings were explained by either decreased preload or increased afterload, or a combination of both. This result seems to be associated with effect of SARS-CoV-2 infection-related inflammation on fetal circulation [10]. When RSI patients were grouped according to disease severity, $\mathrm{CCO}$ and z scores decreased significantly in the severe disease group. It has been shown in previous studies that inflammatory cytokine release is associated with disease severity $[29,30]$. The fact that the decrease in CCO could not be detected in the general RSI group while it was only observed in the severe disease group might be related to this excessive inflammatory response in severe group. Also, when we observed adverse pregnancy outcomes including preterm delivery, fetal distress, NICU admission were significantly higher in severe disease group than the control group. Decreasing fetal cardiac output may have contributed to these adverse pregnancy outcomes.

$\mathrm{CO}$ was found to be significantly associated with advancing gestational age and estimated fetal weight (EFW) [16]. Pilania et al found that $\mathrm{CO}$ is higher in fetuses of diabetic mothers, but they did not adjust the CO for EFW [13]. On the contrary, Winter et al. observed a decrease fetal CO in diabetic mother's fetuses when they include fetal EFW to the calculation and they suggest EFW- corrected CO's are valuable for accurate assessment of fetal cardiac function than $\mathrm{CO}(\mathrm{mL} / \mathrm{min})$ alone [9]. In our study, $\mathrm{CCO}(\mathrm{mL} / \mathrm{min})$ measurement without including fetal weight was found similar between all mild, moderate, severe, and control groups. On the other hand, fetal weight-normalized $\mathrm{CCO}(\mathrm{mL} / \mathrm{min} / \mathrm{kg})$ and $\mathrm{CCO}$ z score were found to be significantly lower in the severe disease group compared to the control and mild disease groups. This result highlights the importance of standardization according to fetal weight and z scores. Non-normalized cardiac morphological measurements and flow measurements in the growing fetus do not appear to be as significant as $\mathrm{Z}$ scores.

The main strength of the study is its novelty, prospective design and evaluation of subgroups according to disease severity. On the other hand, relatively few cases especially in the severe disease group are the main limitation.

\section{Conclusion}

In conclusion, SARS-CoV-2 infections have been conducted on fetal adverse effects $[3,4,6]$. The negative 
impact on placental tissues and fetal organs has been observed [31,32]. In the present study, we showed a decrease in fetal cardiac output, especially in severe diseases, and this is the first study in the literature to evaluate fetal CO in pregnant women recovering from SARS-CoV-2 infection on this view of point. Further studies are needed to clarify the impact of SARS-CoV-2 infection on fetal cardiac function.

Conflicts of interest : None

\section{Tables}

Table 1. Baseline data and characteristics of the groups

Table 2. Clinical characteristics of SARS-CoV-2 infection

Table 3. Fetal echocardiographic findings in RSI and control groups

Table 4. SARS-CoV-2 infection severity and fetal echocardiographic findings

Table 5. Perinatal outcomes in mild, moderate, severe disease and control groups

Figure 1. Aortic valve annulus diameter. L: left, R: right, AV annulus: aortic valve annulus, LA: left atrium, LV: left ventricle, RV: right ventricle, Zs-GA: Z score for gestational age.

Figure 2. Pulmonary valve annulus diameter. PV annulus: pulmonary valve annulus, Ao: aorta RA: right atrium, RV: right ventricle, LPA: left pulmonary artery, RPA: right pulmonary artery, Zs-GA: Z score for gestational age.

Figure 3. The aortic valves flow velocity waveforms and velocity time integral (VTI) were obtained from the left ventricular outflow tract (LVOT) views. Cardiac outputs was calculated as VTI $\times \pi$ (Aortic valve diameter $/ 2) 2 \times$ heart rate.

\section{References}

[1] Wang CL, Liu YY, Wu CH, Wang CY, Wang CH, Long CY. Impact of COVID-19 on Pregnancy. International Journal of Medical Sciences 2021;18:763. https://doi.org/10.7150/IJMS.49923.

[2] Nana M, Nelson-Piercy C. COVID-19 in pregnancy. CME: OBSTETRIC MEDICINE Clinical Medicine 2021;21:446-50. https://doi.org/10.7861/clinmed.2021-0503.

[3] Sahin D, Tanacan A, Erol SA, Yucel Yetiskin FD, Besimoglu B, Ozden Tokalioglu E, et al. Management of pregnant women with COVID-19: A tertiary pandemic center experience on 1416 cases. Journal of Medical Virology 2021;94:1074-84. https://doi.org/10.1002/JMV.27423.

[4] Juan J, Gil MM, Rong Z, Zhang Y, Yang H, Poon LC. Effect of coronavirus disease 2019 (COVID-19) on maternal, perinatal and neonatal outcome: systematic review. Ultrasound in Obstetrics \& Gynecology : The Official Journal of the International Society of Ultrasound in Obstetrics and Gynecology 2020;56:15-27. https://doi.org/10.1002/UOG.22088.

[5] Girolamo R di, Khalil A, Alameddine S, D'angelo E, Galliani C, Matarrelli B, et al. Placental histopathology after SARS-CoV-2 infection in pregnancy: a systematic review and meta-analysis 2021. https://doi.org/10.1016/j.

[6] Allotey J, Stallings E, Bonet M, Yap M, Chatterjee S, Kew T, et al. Clinical manifestations, risk factors, and maternal and perinatal outcomes of coronavirus disease 2019 in pregnancy: living systematic review and meta-analysis. BMJ (Clinical Research Ed) 2020;370.https://doi.org/10.1136/BMJ.M3320.

[7] Kenny J, Plappert T, Doubilet P, Salzman D, Sutton MG. Effects of heart rate on ventricular size, stroke volume, and output in the normal human fetus: a prospective Doppler echocardiographic study. Circulation 1987;76:52-8. https://doi.org/10.1161/01.CIR.76.1.52.

[8] Parer JT. Fetal Circulation. The Global Library of Women's Medicine 2009.https://doi.org/10.3843/GLOWM.10194. 
[9] Winter J, Kulkarni A, Craft M, Li L, Hornberger LK, Danford DA, et al. Depressed left and right ventricular cardiac output in fetuses of diabetic mothers. Echo Research and Practice 2018;5:19-26. https://doi.org/10.1530/ERP-17-0063.

[10] Dahlof B. Effect of angiotensin II blockade on cardiac hypertrophy and remodelling: a review. Journal of Human Hypertension 1995;9.

[11] Bond SJ, Harrison MR, Schmidt KG, Silverman NH, Flake AW, Slotnick RN, et al. Death due to high-output cardiac failure in fetal sacrococcygeal teratoma. Journal of Pediatric Surgery 1990;25:1287-91. https://doi.org/10.1016/0022-3468(90)90535-H.

[12] Davis L, Thornburg KL, Giraud GD. The effects of anaemia as a programming agent in the fetal heart. The Journal of Physiology 2005;565:35-41. https://doi.org/10.1113/JPHYSIOL.2004.082388.

[13] Pilania R, Sikka P, Rohit MK, Suri V, Kumar P. Fetal Cardiodynamics by Echocardiography in Insulin Dependent Maternal Diabetes and Its Correlation with Pregnancy Outcome. Journal of Clinical and Diagnostic Research : JCDR 2016;10. https://doi.org/10.7860/JCDR/2016/17993.8079.

[14] Cardiac screening examination of the fetus: guidelines for performing the "basic" and "extended basic" cardiac scan. Ultrasound in Obstetrics \& Gynecology : The Official Journal of the International Society of Ultrasound in Obstetrics and Gynecology 2006;27:107-13. https://doi.org/10.1002/UOG.2677.

[15] van der Mooren K, Barendregt LG, Wladimiroff JW. Fetal atrioventricular and outflow tract flow velocity waveforms during normal second half of pregnancy. American Journal of Obstetrics and Gynecology 1991;165:668-74. https://doi.org/10.1016/0002-9378(91)90306-C.

[16] Mao YK, Zhao BW, Zhou L, Wang B, Chen R, Wang SS. Z-score reference ranges for pulsed-wave Doppler indices of the cardiac outflow tracts in normal fetuses. The International Journal of Cardiovascular Imaging 2019;35:811-25. https://doi.org/10.1007/S10554-018-01517-1.

[17] Gagnon C, Bigras JL, Fouron JC, Dallaire F. Reference Values and Z Scores for Pulsed-Wave Doppler and M-Mode Measurements in Fetal Echocardiography. Journal of the American Society of Echocardiography : Official Publication of the American Society of Echocardiography 2016;29:448-460.e9. https://doi.org/10.1016/J.ECHO.2016.01.002.

[18] Hadlock FP, Harrist RB, Carpenter RJ, Deter RL, Park SK. Sonographic estimation of fetal weight. The value of femur length in addition to head and abdomen measurements. Radiology 1984;150:535-40. https://doi.org/10.1148/RADIOLOGY.150.2.6691115.

[19] de Terwangne C, Laouni J, Jouffe L, Lechien JR, Bouillon V, Place S, et al. Predictive Accuracy of COVID-19 World Health Organization (WHO) Severity Classification and Comparison with a Bayesian-Method-Based Severity Score (EPI-SCORE). Pathogens (Basel, Switzerland) 2020;9:1-17. https://doi.org/10.3390/PATHOGENS9110880.

[20] Kreis NN, Ritter A, Louwen F, Yuan J. A Message from the Human Placenta: Structural and Immunomodulatory Defense against SARS-CoV-2. Cells 2020;9. https://doi.org/10.3390/CELLS9081777.

[21] Sharps MC, Hayes DJL, Lee S, Zou Z, Brady CA, Almoghrabi Y, et al. A structured review of placental morphology and histopathological lesions associated with SARS-CoV-2 infection. Placenta 2020;101:13-29. https://doi.org/10.1016/J.PLACENTA.2020.08.018.

[22] Seymen CM. Being pregnant in the COVID-19 pandemic: Effects on the placenta in all aspects. Journal of Medical Virology 2021;93:2769-73. https://doi.org/10.1002/JMV.26857.

[23] Ling HZ, Guy GP, Bisquera A, Nicolaides KH, Kametas NA. Maternal cardiac adaptation and fetal growth. American Journal of Obstetrics and Gynecology 2021;224:601.e1-601.e18. https://doi.org/10.1016/J.AJOG.2020.12.1199. 
[24] Huhta JC. Diagnosis and treatment of foetal heart failure: foetal echocardiography and foetal hydrops. Cardiology in the Young 2015;25 Suppl 2:100-6. https://doi.org/10.1017/S104795111500089X.

[25] Vincent JL. Understanding cardiac output. Critical Care (London, England) 2008;12. https://doi.org/10.1186/CC6975.

[26] Statile CJ, Cnota JF, Gomien S, Divanovic A, Crombleholme T, Michelfelder E. Estimated cardiac output and cardiovascular profile score in fetuses with high cardiac output lesions. Ultrasound in Obstetrics \& Gynecology : The Official Journal of the International Society of Ultrasound in Obstetrics and Gynecology 2013;41:54-8. https://doi.org/10.1002/UOG.12309.

[27] Copel JA, Grannum PA, Green JJ, Belanger K, Hanna N, Jaffe CC, et al. Fetal cardiac output in the isoimmunized pregnancy: a pulsed Doppler-echocardiographic study of patients undergoing intravascular intrauterine transfusion. American Journal of Obstetrics and Gynecology 1989;161:361-5. https://doi.org/10.1016/0002-9378(89)90520-6.

[28] Rizzo G, Mattioli C, Mappa I, Bitsadze V, Khizroeva J, Słodki M, et al. Hemodynamic factors associated with fetal cardiac remodeling in late fetal growth restriction: A prospective study. Journal of Perinatal Medicine 2019;47:683-8. https://doi.org/10.1515/JPM-2019-0217/PDF.

[29] Wang J, Jiang M, Chen X, Montaner LJ. Cytokine storm and leukocyte changes in mild versus severe SARS-CoV-2 infection: Review of 3939 COVID-19 patients in China and emerging pathogenesis and therapy concepts. J Leukoc Biol 2020. https://doi.org/10.1002/JLB.3COVR0520-272R.

[30] Zeng F, Huang Y, Guo Y, Yin M, Chen X, Xiao L, et al. Association of inflammatory markers with the severity of COVID-19: A meta-analysis. International Journal of Infectious Diseases : IJID : Official Publication of the International Society for Infectious Diseases 2020;96:467-74. https://doi.org/10.1016/J.IJID.2020.05.055.

[31] Facchetti F, Bugatti M, Drera E, Tripodo C, Sartori E, Cancila V, et al. SARS-CoV2 vertical transmission with adverse effects on the newborn revealed through integrated immunohistochemical, electron microscopy and molecular analyses of Placenta. EBioMedicine 2020;59. https://doi.org/10.1016/J.EBIOM.2020.102951.

[32] Ping Wong Y, Yee Khong T, Chin Tan G. diagnostics The Effects of COVID-19 on Placenta and Pregnancy: What Do We Know So Far? 2021. https://doi.org/10.3390/diagnostics11010094.

Table 1. Baseline data and characteristics of the groups

\begin{tabular}{llll}
\hline & Control $(\mathbf{n = 5 0 )}$ & RSI $(\mathbf{n = 4 8 )}$ & P-Values \\
\hline Maternal age & $27 \pm 5$ & $28 \pm 4$ & 0,200 \\
BMI & $27,2 \pm 4,7$ & $29,0 \pm 4,7$ & 0,062 \\
Nulliparity & $18(51,4 \%)$ & $17(48,6 \%)$ & 0,952 \\
Gestational age at ultrasound assessment (week) & $34(2,6)$ & $32(7,6)$ & 0,565 \\
\hline
\end{tabular}

Data given as median (interquartile range); number, percentile (n,\%). RSI: Recovery SARS-CoV-2 Infection, BMI: Body mass index

Table 2. Clinical characteristics of SARS-CoV-2 infection

Variables

Gestational age at diagnosis (week)

Severity of disease Mild disease Moderate disease Severe disease Critically severe disease

Admission to hospital ( $\mathrm{n}, \%)$

ICU admission 
Variables

COVİD-19 therapy (n,\%) Low molecular weight heparin (n,\%) Lopinavir-ritonavir (n,\%) Glucocorticoids (n Respiratory support (n, \%) Nasal oxygen therapy (n, \%) Oxygen mask with reservoir bag (n, \%)

Data given as median (interquartile range), max-min value, number, percentile (n,\%). RSI: Recovery SARS-

CoV-2 Infection, ICU: intensive care unit.

Table 3. Fetal echocardiographic findings in RSI and control groups

\begin{tabular}{|c|c|c|c|}
\hline & Control $(n=50)$ & RSI $(n=48)$ & P-Values \\
\hline Heart rate & $140 \pm 12$ & $136 \pm 9$ & 0,248 \\
\hline Aortic annulus (cm) & $5,5 \pm 0,9$ & $5,2 \pm 0,9$ & 0,115 \\
\hline PA annulus $(\mathrm{cm})$ & $6,4 \pm 1,2$ & $6,1 \pm 1,2$ & 0,087 \\
\hline Aortic VTI (cm) & $0,083 \pm 0,015$ & $0,079 \pm 0,015$ & 0,326 \\
\hline PA VTI (cm) & $0,082 \pm 0,020$ & $0,097 \pm 0,116$ & 0,849 \\
\hline $\mathrm{LCO}(\mathrm{mL} / \mathrm{min})$ & $285,1 \pm 114,7$ & $246,7 \pm 109,0$ & 0,058 \\
\hline LCO z score & $-0,4 \pm 1$ & $-0,8 \pm 0,9$ & 0,041 \\
\hline $\operatorname{RCO}(\mathrm{mL} / \mathrm{min})$ & $395,0 \pm 193,6$ & $340,5 \pm 137,5$ & 0,243 \\
\hline RCO z score & $-0,8 \pm 1,3$ & $-1,3 \pm 1$ & 0,121 \\
\hline $\mathrm{CCO}(\mathrm{mL} / \mathrm{min})$ & $681,0 \pm 289,6$ & $585,9 \pm 225,5$ & 0,168 \\
\hline CCO z score & $-0,6 \pm 0,8$ & $-1,0 \pm 0,6$ & 0,058 \\
\hline $\mathrm{CCO}(\mathrm{mL} / \mathrm{min} / \mathrm{kg})$ & $352,6 \pm 119,9$ & $328,9 \pm 108,3$ & 0,365 \\
\hline $\mathrm{RCO} / \mathrm{LCO}$ ratio & $1,37 \pm 0,4$ & $1,46 \pm 0,5$ & 0,726 \\
\hline
\end{tabular}

Data given as mean \pm SD. PA: pulmonary artery, VTI: velocity time integral, LCO: left cardiac output, RCO: right cardiac output, and CCO: combined cardiac output.

Table 4. SARS-CoV-2 infection severity and fetal echocardiographic findings

\begin{tabular}{lllll}
\hline & Control $(\mathbf{n}=\mathbf{5 0})$ & Mild infection $\mathbf{( n = 1 8 )}$ & Moderate infection $\mathbf{( n = 2 0 )}$ & Severe infectio \\
\hline LCO (mL/min) & $282,2 \pm 114,4$ & $268,6 \pm 102,4$ & $248,1 \pm 111,5$ & $204,9 \pm 114,5$ \\
LCO z score & $-0,4 \pm 1$ & $-0,6 \pm 0,6$ & $-0,7 \pm 0,8$ & $-1,5 \pm 1,3^{* * *}$ \\
RCO (mL/min) & $385,6 \pm 185,2$ & $368,0 \pm 147,7$ & $320,9 \pm 123,5$ & $330,2 \pm 151,2$ \\
RCO z score & $-0,9 \pm 1,2$ & $-1,1 \pm 0,7$ & $-1,2 \pm 1,3$ & $-1,8 \pm 0,9$ \\
CCO (mL/min) & $668,7 \pm 280,8$ & $637,0 \pm 240,9$ & $568,6 \pm 208,5$ & $528,7 \pm 233,4$ \\
CCO $\mathbf{~ s c o r e ~}$ & $-0,7 \pm 0,7$ & $-0,8 \pm 0,4$ & $-0,9 \pm 0,6$ & $-1,5 \pm 0,7^{* * *}$ \\
CCO (mL/min/kg) & $361,3 \pm 115,3$ & $366,1 \pm 105$ & $331,3 \pm 96,1$ & $257,3 \pm 111,9^{* *}$ \\
\hline
\end{tabular}

Data given as mean \pm SD. LCO: left cardiac output, RCO: right cardiac output, and CCO: combined cardiac output. ${ }^{* *}$ Severe disease was significantly different with control and mild disease, ${ }^{* * *}$ Severe disease was significantly different with control, mild and moderate disease.

Table 5. Perinatal outcomes in mild, moderate, severe disease and control groups

\begin{tabular}{lllll}
\hline & Control $(\mathbf{n}=\mathbf{5 0})$ & Mild infection $(\mathbf{n}=\mathbf{1 8})$ & Moderate infection $(\mathbf{n}=\mathbf{2 0})$ & Se \\
\hline Gestational age at birth (week) & $37,3 \pm 2,2$ & $36,1 \pm 4,5$ & $37,2 \pm 2,6$ & 33 \\
Birth weight (g) & $3049 \pm 474$ & $3067 \pm 651$ & $3032 \pm 405$ & 23 \\
Preterm delivery rate (n,\%) & $2(4 \%)$ & $3(16,7 \%)$ & $2(10 \%)$ & 4
\end{tabular}




\begin{tabular}{lllll}
\hline & Control $(\mathbf{n}=\mathbf{5 0})$ & Mild infection $(\mathbf{n = 1 8})$ & Moderate infection $(\mathbf{n = 2 0})$ & Se \\
\hline Fetal growth retardation $(\mathbf{n}, \%)$ & - & $1(5,6 \%)$ & $1(5 \%)$ & 1 \\
Fetal distress (n,\%) & $1(2 \%)$ & $1(5,6 \%)$ & $1(5 \%)$ & 3 \\
1st minApgar $<\mathbf{7}$ & $2(4 \%)$ & $2(11,1 \%)$ & $3(15 \%)$ & 3 \\
5st min Apgar <7 & - & - & $1(5 \%)$ & 1 \\
Hospitalization in NICU (n,\%) & $2(4 \%)$ & $2(11,1 \%)$ & $4(20 \%)$ & 6 \\
\hline
\end{tabular}

Data given as median (interquartile range); number, percentile (n,\%). C/S: cesarian section, NICU: Neonatal intensive care unit. * Severe disease was significantly different with control, ${ }^{* * *}$ Severe disease was significantly different with control, mild and moderate disease.

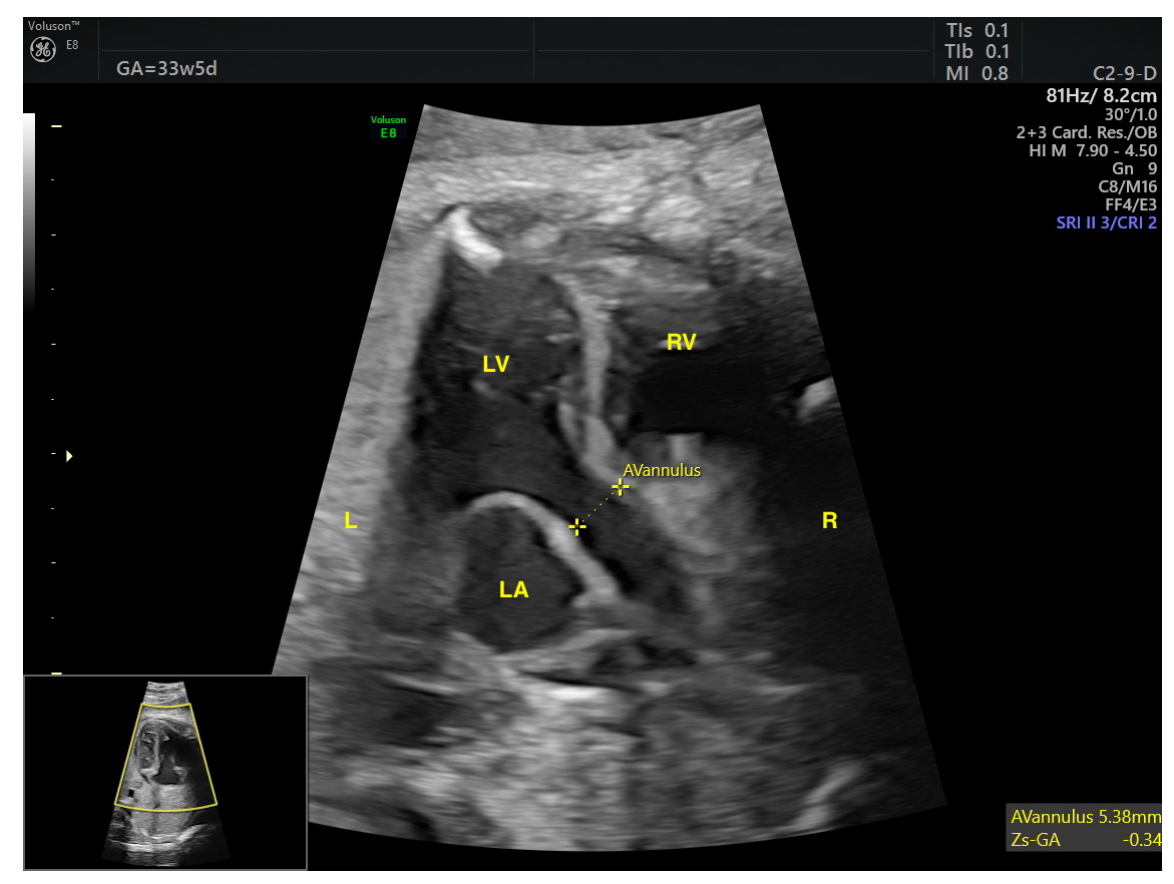



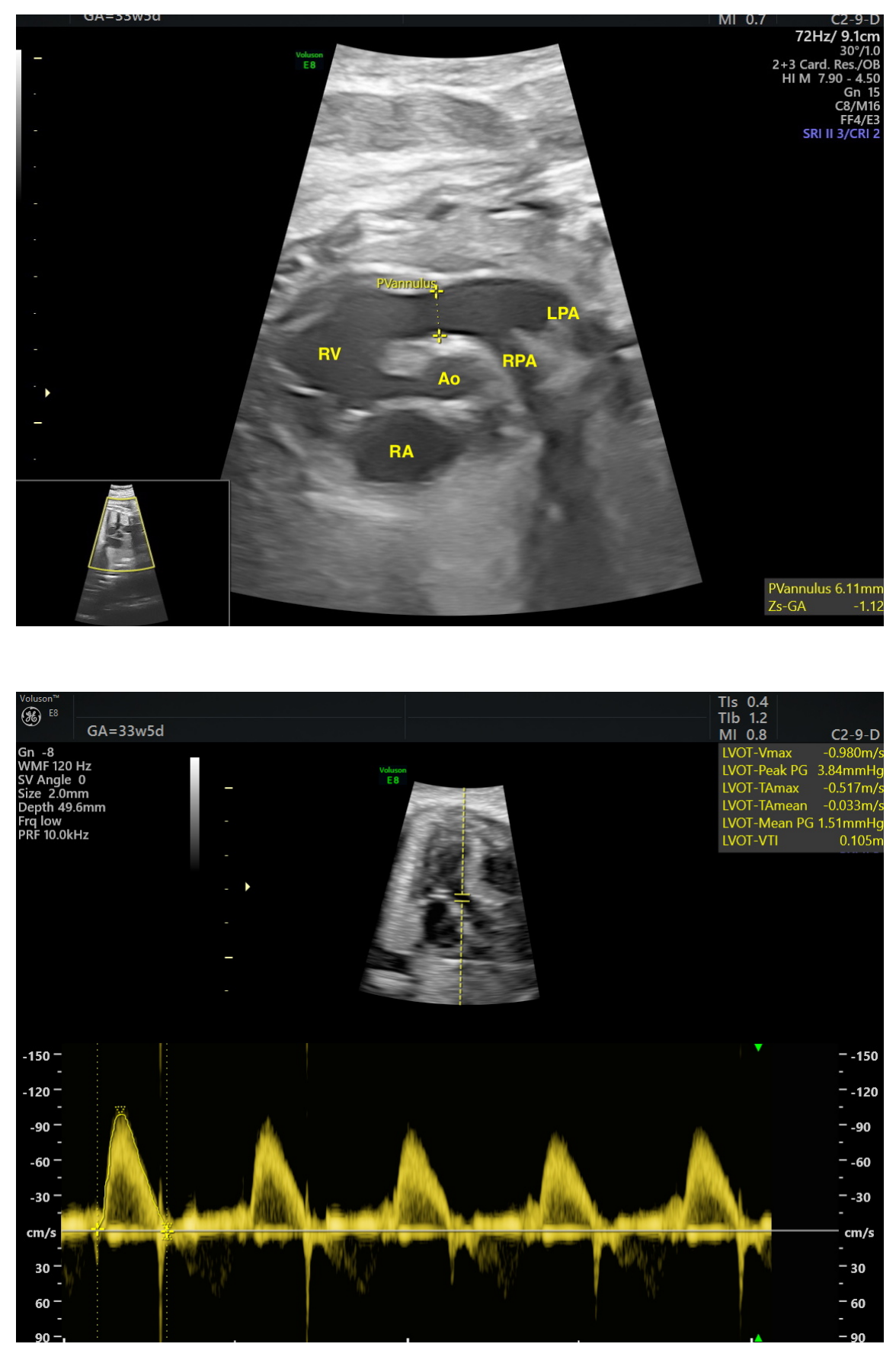